\title{
Effect of Combinations of Monochromatic LED Light Color on the Performance and Behavior of Laying Hens
}

\author{
Md. Rakibul Hassan ${ }^{1}$, Shabiha Sultana ${ }^{1}$, Ho S. Choe ${ }^{2}$ and Kyeong S. Ryu ${ }^{1}$ \\ ${ }^{1}$ Department of Animal Science, College of Agricultural Life and Science, \\ Chonbuk National University, Jeonju, 561-756, Republic of Korea \\ ${ }^{2}$ Department of Animal Biotechnology, College of Agricultural Life and Science, \\ Chonbuk National University, Jeonju, 561-756, Republic of Korea
}

\begin{abstract}
The present study evaluated the effect of monochromatic and combinations of light emitting diode light color on the performance and behavior of laying hens. At 12 weeks of age, $600 \mathrm{Hy}$-line Brown pullets were randomly divided into eight lighting treatments with three replicates of 25 birds in each room. The lightning was set-up as follows; white $(\mathrm{W}$, control; $16 \mathrm{~h} / \mathrm{d})$, red $(\mathrm{R} ; 618-635 \mathrm{~nm} ; 16 \mathrm{~h} / \mathrm{d})$, green $(\mathrm{G} ; 515-535 \mathrm{~nm} ; 16 \mathrm{~h} / \mathrm{d})$, blue $(\mathrm{B} ; 455-470 \mathrm{~nm} ; 16$ $\mathrm{h} / \mathrm{d})$, red $\rightarrow$ green $(\mathrm{R} \rightarrow \mathrm{G} ; 14 \mathrm{~h} \rightarrow 2 \mathrm{~h} / \mathrm{d})$, red $\rightarrow$ blue $(\mathrm{R} \rightarrow \mathrm{B} ; 14 \mathrm{~h} \rightarrow 2 \mathrm{~h} / \mathrm{d})$, red $\rightarrow$ green $\rightarrow$ blue $(\mathrm{R} \rightarrow \mathrm{G} \rightarrow \mathrm{B} ; 12 \mathrm{~h} \rightarrow 2 \mathrm{~h} \rightarrow 2$ $\mathrm{h} / \mathrm{d})$ and red $\rightarrow$ blue $\rightarrow$ green $(\mathrm{R} \rightarrow \mathrm{B} \rightarrow \mathrm{G} ; 12 \mathrm{~h} \rightarrow 2 \mathrm{~h} \rightarrow 2 \mathrm{~h} / \mathrm{d})$ light treatments. The light colors significantly influenced egg production, which was higher for the combination $R \rightarrow G(89.56 \%)$ and monochromatic $R(87.34 \%)$ and lower in the G $(85.26 \%)$ and B $(83.75 \%)$ light treatments. Conversely, egg weight was remarkably heavier in the B and G light treatments than those of monochromatic $\mathrm{R}$ and combination treatments. Concurrently, better feed conversion ratio was found for the combination $R \rightarrow G \rightarrow B$ and $R \rightarrow G$ and the monochromatic $R$ light treatment. Egg shells were significantly pigmented by the combinations of $R \rightarrow B \rightarrow G$ and $R \rightarrow G \rightarrow B$. Furthermore, egg shell breaking strength increased following the $\mathrm{G}$ treatment. Higher frequencies of ground pecking, ground scratching, and tail wagging were observed in the $\mathrm{R}$ group and these behaviors were less frequent in the B groups. Birds under $\mathrm{G}$ and $\mathrm{B}$ lights spent a longer time perching. These results suggest that a combination of $R \rightarrow G$ and monochromatic $R$ light enhanced egg production. In contrast, $\mathrm{R}$ light activated the bird's movement, whereas B light decreased movement, and birds spent a longer time perching.
\end{abstract}

Key words: laying hens, LED light color, performance and behavior

J. Poult. Sci., 51: 321-326, 2014

\section{Introduction}

Many lighting systems (mercury vapor lamps, incandescent, fluorescent, compact fluorescent, fluorescent tube and high intensity discharge lighting, UV, and filtered light) have been used in the poultry industry. Light-emitting diode (LED) lamps have recently been introduced into the commercial market and poultry farmers have shown growing interest, as these lamps reduce power consumption and thus reducing rearing cost of laying hens (Rozenboim et al., 1998). Therefore, Europe banned almost all incandescent lighting in 2012. The Korean government introduced a new energy-saving target of achieving 100\% LED lighting by public utilities in 2020. LED light colors have received con-

Received: June 2, 2013, Accepted: October 6, 2013

Released Online Advance Publication: November 25, 2013

Correspondence: Prof. K. S. Ryu PhD, Department of Animal Science, College of Agriculture and Life Science, Chonbuk National University, Jeonju 561-756 Republic of Korea. (E-mail: seon@jbnu.ac.kr) siderable attention as alternatives to conventional lighting in the poultry industry. However, most researchers have focused on monochromatic LED light color and found that red (R) light enhances egg production of laying hens (Rozenboim et al., 1998; Kim et al., 2012), whereas hens lay larger eggs under blue (B) or green (G) light (Er et al., 2007).

A recent study using combinations of light color on broiler breeder hens indicated that extra-retinal photo-stimulation with $\mathrm{R}$ light increases reproductive performance, whereas retinal photo-stimulation with $\mathrm{G}$ light combined with nonphotostimulatory $\mathrm{R}$ light inhibits reproduction in broiler breeder hens (Mobarkey et al., 2010). But, little is known about the combinations $\mathrm{R} \rightarrow \mathrm{G} \rightarrow \mathrm{B}(12 \mathrm{~h} \rightarrow 2 \mathrm{~h} \rightarrow 2 \mathrm{~h}$ per day), $\mathrm{R} \rightarrow \mathrm{B} \rightarrow \mathrm{G}(12 \mathrm{~h} \rightarrow 2 \mathrm{~h} \rightarrow 2 \mathrm{~h}$ per day), $\mathrm{R} \rightarrow \mathrm{G}(14 \mathrm{~h} \rightarrow 2 \mathrm{~h}$ per day), and $R \rightarrow B(14 h \rightarrow 2 h$ per day) on the performance and behavior of laying hens. Furthermore, behavior is not commonly monitored in colored lighting research. Therefore, it is important to understand whether and how different combinations of LED light color affect performance and behavior 
of laying hens. The present study was designed to bridge this gap by investigating the effects of various combinations of monochromatic light colors on the performance and behavior of laying hens.

\section{Materials and Methods}

\section{Experimental Design}

This experiment was conducted at the Poultry Experimental Facility of the Department of Animal Science, Chonbuk National University, Republic of Korea. In total, 600 Hy-line brown pullets were obtained from a commercial farm at 12 weeks of age. Upon arrival, the pullets were randomly housed in 24 environmental and lightproof rooms (eight treatments with three replicates each and 25 birds in each room). Pullets from 12 to 21 weeks of age were subjected to a restricted commercial feeding program with daily administration of feed, as recommended by the Hy-line Brown management guide. At the end of 21 weeks, all birds were allowed free access to feed (ME, $2,750 \mathrm{Kcal} / \mathrm{kg}$; CP, $16 \%$; $\mathrm{Ca}, 3.9 \%$; and $\mathrm{AP}, 0.275 \%$ ) and water throughout the study, which continued for 48 weeks. In a completely randomized design, the LED light treatments were 1) R, 618-635 nm, 2) G, 515-535 nm, 3) B, 455-470 nm, 4) control white (W), (fluorescent light), 5) $\mathrm{R} \rightarrow \mathrm{G}$, 6) $\mathrm{R} \rightarrow \mathrm{B}$, 7) $\mathrm{R}$ $\rightarrow \mathrm{G} \rightarrow \mathrm{B}$, and 8) $\mathrm{R} \rightarrow \mathrm{B} \rightarrow \mathrm{G}$ combination treatments. The

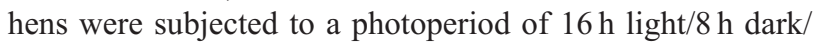
day. Light intensity was measured as $\mathrm{W} / \mathrm{m}^{2}$ of irradiance using a radiometer in each cell at seven constant locations at bird's eye level, as birds receive light by retinal and extraretinal photoreceptors. Thereafter, irradiance was converted to illuminance so that average light intensity was $20 \pm$ 0.21 lux in each compartment, which enabled the birds to receive the same amount of light. Lighting was on from 6 AM to 10 PM daily in the monochromatic R, G, B, and W treatments. Therefore, $\mathrm{R}$ light was provided $14 \mathrm{~h}(6.00 \mathrm{AM}$ to $8.00 \mathrm{PM}$ ) in the $\mathrm{R} \rightarrow \mathrm{G}$ treatment and then moved to $\mathrm{G}$ light for $2 \mathrm{~h}$ (8.00 PM to $10.00 \mathrm{PM}$ ) daily. In the case of the $\mathrm{R} \rightarrow \mathrm{B}$ treatment, $\mathrm{R}$ light was provided for $14 \mathrm{~h}(6.00 \mathrm{AM}$ to 8.00 $\mathrm{PM})$ and then moved to B light for $2 \mathrm{~h}$ (8.00 PM to 10.00 PM) daily. In contrast, in the $R \rightarrow G \rightarrow B$ treatment, $R$ light was provided $12 \mathrm{~h}(6.00 \mathrm{AM}$ to $6.00 \mathrm{PM})$ and then G light was provided for $2 \mathrm{~h}(6.00 \mathrm{PM}$ to $8.00 \mathrm{PM})$ and again B light was provided for $2 \mathrm{~h}$ (8.00 PM to $10.00 \mathrm{PM})$ daily. Thus, the $\mathrm{R} \rightarrow \mathrm{B} \rightarrow \mathrm{G}$ light treatment was provided consecutively as $\mathrm{R} \rightarrow$ $\mathrm{G} \rightarrow \mathrm{B}$, daily. These light colors were changed by an automatic lighting software program. The LED light plate $(38 \times$ $24 \mathrm{~cm}$; Taejong C \& I Co., Seoul, Korea) contained six lines and each line had 12, 8, and 7 small LED bulbs for the R, G, and $\mathrm{B}$ lights, respectively. The lights were hung $2 \mathrm{~m}$ over the floor litter. The LED plates were pointed in a downward direction to light the pens. The electric voltage of the R, G, and B bulb devices was 2.0, 3.2, and 3.2 volts, respectively. The LED bulb devices were forwarded the same current of $\mathrm{I}_{\mathrm{F}}$ $=60 \mathrm{~mA}$. The chicks were housed in separate lightproof rooms $\left(5 \mathrm{~m}^{2}\right)$ equipped with a ventilation fan in the roof of the central arena that circulated fresh air inside the pen. Air temperature $\left(21-24^{\circ} \mathrm{C}\right)$ and humidity $(65-70 \%)$ were mea- sured inside each pen to ensure that any preferences in each pen were due to lighting and not due to temperature or humidity.

\section{Performance and Egg Quality of the Laying Hens}

Egg production, egg weight, and feed consumption in each treatment were recorded daily. Consequently, egg mass ( $\%$ of egg production $\times$ egg weight $/ 100$ ) and feed conversion ( $g$ of feed/g of egg) were calculated based on egg production, egg weight, and feed consumption. At the end of the experiment, 30 eggs were randomly picked from each experimental group to assess egg quality parameters. Egg shell breaking strength was measured with an egg multi-tester instrument (QC-SPA, TSS) and expressed as units of compressive force exposed to the unit egg shell surface area $\left(\mathrm{kg} / \mathrm{cm}^{2}\right)$. Then, the eggs were singularly weighed and carefully broken on a glass plate, and egg shell color, albumin height, Haugh unit, and yolk color were measured using egg quality equipment (QCM+-System, TSS).

\section{Behavior of Laying Hens}

Behavioral observations were conducted when the birds were 29-36 weeks of age. The video of laying hens was recorded for 3 consecutive days/week. The location and behavior of the birds were recorded at the same time for $5 \mathrm{~h}$ per day, $2 \mathrm{~h}$ in the morning $(9.30-11.30 \mathrm{AM})$ and $3 \mathrm{~h}$ in the afternoon $(5.30-8.30 \mathrm{PM})$. The $3 \mathrm{~h}$ in the afternoon were used due to the change in the light color at 6.00 and $8.00 \mathrm{PM}$ daily. A camera (mini U8, DAMOA, Seoul, Korea) captured a full view of the pen. The recorded video was analyzed at 5 minute intervals (Prayitno et al., 1997), and the frequencies of these behaviors were recorded. Behaviors were recorded for the following activities: feather pecking, preening, perching, dust-bathing, ground pecking, ground scratching, tail-wagging, and wing-flapping. Feather pecking, preening, dust bathing, ground pecking, ground scratching, tail wagging, and wing flapping behaviors were expressed as a frequency, and perching behavior was expressed as time/h.

A bird was considered to be feather pecking when it was investigating another bird's feathers using the beak. Preening was considered when plumage was manipulated with the beak. A bird was considered to be "dust-bathing" when they scratched the floor and sprinkled their body with litter, and shook their legs quickly. We recorded "perching" when a bird was located over floor level. When a bird pecked the ground but not in or around the feeder it was counted as ground pecking behavior. Extending both wings out from the body simultaneously and flapping was considered wing flapping. Ground scratching was recorded when birds scratched the litter with their claws. Tail wagging was characterized by depressed tail feathers that were moving rapidly from side to side.

\section{Statistical Analysis}

A one-way analysis of variance was used to compare means of the groups exposed to the different lighting treatments. Behavioral data from 10 chicks per pen were averaged; thus, each pen produced one data point for each behavior both in the morning and afternoon. Because the data of each pen observation were linked, 10 birds per 
Table 1. Effect of LED color on the performance (22-48 weeks) of laying hens

\begin{tabular}{|c|c|c|c|c|c|c|c|c|c|c|}
\hline \multirow{2}{*}{ Parameter } & \multicolumn{8}{|c|}{ Treatment } & \multirow{2}{*}{ SEM } & \multirow{2}{*}{$P$ value } \\
\hline & $\mathrm{R}$ & G & $\mathrm{B}$ & W & $\mathrm{R} \rightarrow \mathrm{G}$ & $\mathrm{R} \rightarrow \mathrm{B}$ & $\mathrm{R} \rightarrow \mathrm{G} \rightarrow \mathrm{B}$ & $\mathrm{R} \rightarrow \mathrm{B} \rightarrow \mathrm{G}$ & & \\
\hline \multicolumn{11}{|c|}{ Number of egg production per hen } \\
\hline $22-30 \mathrm{wk}$ & $44.37^{\mathrm{abc}}$ & $43.11^{\mathrm{bcd}}$ & $42.21^{\mathrm{d}}$ & $42.98^{\mathrm{cd}}$ & $45.41^{\mathrm{a}}$ & $44.07^{\mathrm{abc}}$ & $43.99^{\mathrm{abc}}$ & $44.67^{\mathrm{ab}}$ & 0.25 & 0.01 \\
\hline $31-39 \mathrm{wk}$ & $52.78^{\mathrm{ab}}$ & $51.66^{\mathrm{cd}}$ & $51.09^{\mathrm{d}}$ & $51.95^{\mathrm{bcd}}$ & $53.19^{\mathrm{a}}$ & $52.11^{\mathrm{bc}}$ & $53.10^{\mathrm{a}}$ & $52.31^{\mathrm{abc}}$ & 0.16 & 0.002 \\
\hline $40-48$ wk & $53.11^{\mathrm{ab}}$ & $51.93^{\mathrm{c}}$ & $50.79^{\mathrm{d}}$ & $52.12^{\mathrm{bc}}$ & $53.47^{\mathrm{a}}$ & $52.23^{\mathrm{bc}}$ & $53.41^{\mathrm{a}}$ & $52.30^{\mathrm{bc}}$ & 0.19 & 0.002 \\
\hline Total & $150.36^{\mathrm{ab}}$ & $146.69^{\mathrm{d}}$ & $144.09^{\mathrm{d}}$ & $147.04^{\mathrm{cd}}$ & $152.06^{\mathrm{a}}$ & $148.40^{\mathrm{bcd}}$ & $150.51^{\mathrm{ab}}$ & $149.27^{\mathrm{bc}}$ & 0.54 & 0.001 \\
\hline \multicolumn{11}{|c|}{ Egg production $(\%)$} \\
\hline $22-30 \mathrm{wk}$ & $77.84^{\mathrm{abc}}$ & $75.63^{\mathrm{bcd}}$ & $74.05^{\mathrm{d}}$ & $75.39^{\mathrm{cd}}$ & $79.66^{\mathrm{a}}$ & $77.31^{\mathrm{abc}}$ & $77.18^{\mathrm{abc}}$ & $78.36^{\mathrm{ab}}$ & 0.43 & 0.01 \\
\hline $31-39 w k$ & $92.59^{\mathrm{ab}}$ & $90.63^{\mathrm{cd}}$ & $89.64^{\mathrm{d}}$ & $91.14^{\mathrm{bcd}}$ & $93.31^{\mathrm{a}}$ & $91.41^{\mathrm{bc}}$ & $93.16^{\mathrm{a}}$ & $91.76^{\mathrm{abc}}$ & 0.29 & 0.002 \\
\hline $40-48$ wk & $91.57^{\mathrm{ab}}$ & $89.54^{\mathrm{c}}$ & $87.57^{\mathrm{d}}$ & $89.86^{\mathrm{bc}}$ & $92.19^{\mathrm{a}}$ & $90.05^{\mathrm{bc}}$ & $92.09^{\mathrm{a}}$ & $90.17^{\mathrm{bc}}$ & 0.33 & 0.002 \\
\hline Mean & $87.34^{\mathrm{ab}}$ & $85.26^{\mathrm{bc}}$ & $83.75^{\mathrm{c}}$ & $85.46^{\mathrm{bc}}$ & $89.56^{\mathrm{a}}$ & $86.26^{\mathrm{b}}$ & $87.48^{\mathrm{ab}}$ & $86.76^{\mathrm{b}}$ & 0.41 & 0.002 \\
\hline \multicolumn{11}{|c|}{ Egg weight $(\mathrm{g})$} \\
\hline $22-30 \mathrm{wk}$ & $60.01^{\mathrm{bc}}$ & $60.86^{\mathrm{ab}}$ & $61.87^{\mathrm{a}}$ & $59.85^{\mathrm{bc}}$ & $59.57^{\mathrm{bc}}$ & $59.09^{\mathrm{c}}$ & $60.62^{\mathrm{abc}}$ & $60.35^{\mathrm{abc}}$ & 0.22 & 0.02 \\
\hline $31-39 w k$ & $62.47^{\mathrm{bc}}$ & $62.37^{\mathrm{ab}}$ & $63.64^{\mathrm{a}}$ & $62.34^{\mathrm{bc}}$ & $63.01^{\mathrm{abc}}$ & $61.96^{\mathrm{c}}$ & $62.80^{\mathrm{abc}}$ & $62.47^{\mathrm{bc}}$ & 0.14 & 0.03 \\
\hline $40-48 \mathrm{wk}$ & $62.79^{\mathrm{b}}$ & $64.94^{\mathrm{a}}$ & $65.39^{\mathrm{a}}$ & $63.35^{\mathrm{b}}$ & $63.20^{\mathrm{b}}$ & $62.69^{\mathrm{b}}$ & $62.88^{\mathrm{b}}$ & $62.56^{\mathrm{b}}$ & 0.34 & 0.003 \\
\hline Mean & $61.76^{\mathrm{b}}$ & $63.06^{\mathrm{a}}$ & $63.63^{\mathrm{a}}$ & $61.85^{\mathrm{b}}$ & $61.93^{\mathrm{b}}$ & $61.25^{\mathrm{b}}$ & $62.10^{\mathrm{b}}$ & $61.79^{\mathrm{b}}$ & 0.17 & 0.004 \\
\hline \multicolumn{11}{|c|}{ Egg mass $(\mathrm{g})$} \\
\hline $22-30 \mathrm{wk}$ & 46.72 & 46.03 & 45.81 & 45.12 & 47.46 & 45.69 & 46.79 & 47.30 & 0.27 & 0.32 \\
\hline 31-39 wk & 57.86 & 57.43 & 57.04 & 56.82 & 58.79 & 56.64 & 58.51 & 57.32 & 0.21 & 0.07 \\
\hline $40-48$ wk & 57.49 & 58.15 & 57.27 & 56.92 & 58.26 & 56.45 & 57.91 & 56.42 & 0.19 & 0.08 \\
\hline Mean & 53.94 & 53.77 & 53.29 & 52.81 & 50.37 & 52.83 & 54.32 & 53.62 & 0.49 & 0.66 \\
\hline
\end{tabular}

SEM, standard error; ${ }^{a, b, c, d}$ Values with the same letters in the column are not significantly different at the 5\% level; R, red; G, green; B, blue; W, white; $\mathrm{R} \rightarrow \mathrm{G}$, red $\rightarrow$ green; $\mathrm{R} \rightarrow \mathrm{B}$, red $\rightarrow$ blue; $\mathrm{R} \rightarrow \mathrm{G} \rightarrow \mathrm{B}$, red $\rightarrow$ green $\rightarrow$ blue; $\mathrm{R} \rightarrow \mathrm{B} \rightarrow \mathrm{G}$, red $\rightarrow$ blue $\rightarrow$ green.

lighting pen, 5 days/week for 5 weeks with two replicate pens were used to measure each variable. Therefore, mean data from 25 birds for each behavior with two replications were analyzed by the nonparametric one-way Proc Univariate test in SAS (SAS ${ }^{\circledR}$ 9.1., Cary, NC, USA). When significant differences were found, Duncan's new multiplerange test was performed (Steel and Torrie, 1980). A pvalue $<0.05$ was considered significant.

\section{Results}

\section{Production Parameters}

After 12-48 weeks of light exposure, monochromatic R light increased egg production of laying hens more than that of the $\mathrm{G}$ and $\mathrm{B}$ treatments, whereas $\mathrm{W}$ color had an intermediate effect (Table 2). Therefore, light switched from monochromatic $\mathrm{R}$ to the combination $\mathrm{R} \rightarrow \mathrm{G}$ treatment increased average egg production from 87.34 to $89.56 \%$, but lighting switched from monochromatic $\mathrm{R}$ to the combination $\mathrm{R} \rightarrow \mathrm{B}$ treatment decreased average egg production from 87.34 to $86.26 \%$. However, a further switch from $\mathrm{R} \rightarrow \mathrm{G}$ to either $\mathrm{R} \rightarrow \mathrm{G} \rightarrow \mathrm{B}$ or $\mathrm{R} \rightarrow \mathrm{B} \rightarrow \mathrm{G}$ had no additional effects on egg production. Thereafter, egg weight was influenced ( $p<$ 0.05 ) by light color, in which consistently (22-48 wks) higher egg weight was obtained under B (63.63 g) and G $(63.06 \mathrm{~g})$ light than that under $\mathrm{R}(61.76 \mathrm{~g})$ light and the combinations. However the combination of either two colors $(\mathrm{R} \rightarrow \mathrm{G}$ or $\mathrm{R} \rightarrow \mathrm{B})$ or three colors $(\mathrm{R} \rightarrow \mathrm{G} \rightarrow \mathrm{B}, \mathrm{R} \rightarrow \mathrm{B} \rightarrow \mathrm{G})$ did not influence egg weight of laying hens. In contrast, egg mass tended to increase in the $\mathrm{R} \rightarrow \mathrm{G}$ treatment compared to that in the other monochromatic and combination treatments.
Though feed intake was not influenced by light color, better feed conversion efficiency was found in the $R \rightarrow G$ light treatment than that in the $\mathrm{B}$ and $\mathrm{W}$ treatments.

The present results showed that egg shell color was darker $(p<0.05)$ when birds were exposed to combined light colors $(\mathrm{R} \rightarrow \mathrm{B} \rightarrow \mathrm{G}, \mathrm{R} \rightarrow \mathrm{B}$ and $\mathrm{R} \rightarrow \mathrm{G} \rightarrow \mathrm{B}$ ) than that of monochromatic light. Egg shell breaking strength increased significantly $(p<0.05)$ under the monochromatic $\mathrm{G}$ light compared with that of monochromatic $\mathrm{R}$ and their combined treatment (Table 3). However, no differences were found among the monochromatic and combined lighting colors with respect to albumin height, Haugh units, or yolk color.

\section{Behavior}

Feather pecking, preening, and dust bathing behaviors were not affected by the lighting treatments, but perching behavior increased $(p<0.05)$ under the B light treatment (Table 4). The mean frequency of feather pecking was found under R light, which was lower in the monochromatic B and combined $\mathrm{R} \rightarrow \mathrm{B}$ lighting groups. The other monochromatic lights and their combinations had an intermediate effect. Preening behavior tended to increase under the $R$ and $R \rightarrow G$ lighting treatments. When light color was changed from $\mathrm{R}$ to $\mathrm{B}$ perching behavior increased significantly from 17.49 to $22.94 \mathrm{~min} /$ hour in laying hens. Dust bathing behavior was not affected by light color but a higher tendency was found under the $\mathrm{R}$ lighting treatment than that under the B treatment.

Ground pecking, ground scratching, and tail wagging behaviors were affected by light color (Table 5). Monochromatic $\mathrm{R}, \mathrm{G}$, and the combination of $\mathrm{R} \rightarrow \mathrm{G}$ light color 
Table 2. Effect of LED color on the performance (22-48 weeks) of laying hens

\begin{tabular}{|c|c|c|c|c|c|c|c|c|c|c|}
\hline \multirow{2}{*}{ Parameter } & \multicolumn{8}{|c|}{ Treatment } & \multirow{2}{*}{ SEM } & \multirow{2}{*}{$P$ value } \\
\hline & $\mathrm{R}$ & G & B & W & $\mathrm{R} \rightarrow \mathrm{G}$ & $\mathrm{R} \rightarrow \mathrm{B}$ & $\mathrm{R} \rightarrow \mathrm{G} \rightarrow \mathrm{B}$ & $\mathrm{R} \rightarrow \mathrm{B} \rightarrow \mathrm{G}$ & & \\
\hline \multicolumn{11}{|c|}{ Feed intake $(\mathrm{g} / \mathrm{d})$} \\
\hline $22-30 \mathrm{wk}$ & 129.50 & 130.83 & 128.54 & 129.14 & 130.16 & 129.07 & 131.39 & 130.01 & 0.37 & 0.62 \\
\hline $31-39 \mathrm{wk}$ & 132.83 & 132.15 & 132.75 & 131.88 & 132.97 & 131.38 & 132.41 & 130.28 & 0.32 & 0.45 \\
\hline $40-48 \mathrm{wk}$ & 134.45 & 134.85 & 135.56 & 137.64 & 133.87 & 134.82 & 135.67 & 133.81 & 0.36 & 0.14 \\
\hline Mean & 132.26 & 132.61 & 132.28 & 132.89 & 132.33 & 131.76 & 133.16 & 131.37 & 0.20 & 0.46 \\
\hline \multicolumn{11}{|l|}{ FCR } \\
\hline $22-30 \mathrm{wk}$ & 2.77 & 2.84 & 2.81 & 2.86 & 2.74 & 2.83 & 2.81 & 2.75 & 0.02 & 0.47 \\
\hline $31-39$ wk & 2.29 & 2.30 & 2.33 & 2.32 & 2.26 & 2.32 & 2.26 & 2.27 & 0.01 & 0.15 \\
\hline $40-48$ wk & 2.34 & 2.32 & 2.37 & 2.42 & 2.29 & 2.39 & 2.34 & 2.37 & 0.01 & 0.06 \\
\hline Mean & $2.45^{\mathrm{bc}}$ & $2.47^{\mathrm{abc}}$ & $2.48^{\mathrm{ab}}$ & $2.51^{\mathrm{a}}$ & $2.42^{\mathrm{c}}$ & $2.49^{\mathrm{ab}}$ & $2.45^{\mathrm{bc}}$ & $2.45^{\mathrm{bc}}$ & 0.01 & 0.04 \\
\hline
\end{tabular}

Table 3. Effect of different LED color on egg quality of laying hens

\begin{tabular}{|c|c|c|c|c|c|c|c|c|c|c|}
\hline \multirow{2}{*}{ Parameter } & \multicolumn{8}{|c|}{ Treatment } & \multirow{2}{*}{ SEM } & \multirow{2}{*}{$\mathrm{P}$ value } \\
\hline & $\mathrm{R}$ & G & B & W & $\mathrm{R} \rightarrow \mathrm{G}$ & $\mathrm{R} \rightarrow \mathrm{B}$ & $\mathrm{R} \rightarrow \mathrm{G} \rightarrow \mathrm{B}$ & $\mathrm{R} \rightarrow \mathrm{B} \rightarrow \mathrm{G}$ & & \\
\hline $\mathrm{SC}$ & $26.24^{\mathrm{b}}$ & $26.43^{\mathrm{b}}$ & $26.33^{\mathrm{b}}$ & $27.03^{\mathrm{b}}$ & $27.02^{\mathrm{b}}$ & $27.47^{\mathrm{ab}}$ & $27.36^{\mathrm{ab}}$ & $29.03^{\mathrm{a}}$ & 0.21 & 0.03 \\
\hline $\mathrm{AH}(\mathrm{mm})$ & 9.92 & 10.16 & 10.01 & 9.48 & 9.23 & 9.28 & 10.11 & 9.37 & 0.11 & 0.13 \\
\hline $\mathrm{HU}$ & 97.65 & 97.96 & 97.23 & 95.11 & 94.21 & 94.76 & 98.45 & 95.32 & 0.54 & 0.32 \\
\hline YC & 6.53 & 6.47 & 6.03 & 6.23 & 6.20 & 6.33 & 6.37 & 6.37 & 0.05 & 0.68 \\
\hline $\operatorname{ESBS}\left(\mathrm{Kg} / \mathrm{cm}^{2}\right)$ & $4.88^{\mathrm{bc}}$ & $5.34^{\mathrm{a}}$ & $5.29^{\mathrm{ab}}$ & $5.00^{\mathrm{abc}}$ & $5.01^{\mathrm{abc}}$ & $4.81^{\mathrm{c}}$ & $4.91^{\mathrm{bc}}$ & $4.82^{\mathrm{c}}$ & 0.04 & 0.04 \\
\hline
\end{tabular}

Table 4. Effect of LED color on different behaviors of laying hens

\begin{tabular}{|c|c|c|c|c|c|c|c|c|c|c|c|c|}
\hline \multirow{2}{*}{ Treatment } & \multicolumn{3}{|c|}{ Feather pecking $($ no/h) } & \multicolumn{3}{|c|}{ Preening (no/h) } & \multicolumn{3}{|c|}{ perching time $(\mathrm{min} / \mathrm{h})$} & \multicolumn{3}{|c|}{ Dustbathing (min/h) } \\
\hline & M & A & Mean & M & A & Mean & M & A & Mean & M & A & Mean \\
\hline $\mathrm{R}$ & 16.65 & 23.10 & 19.87 & 687.75 & 751.14 & 719.45 & $16.97^{\mathrm{b}}$ & $17.63^{\mathrm{c}}$ & $17.49^{\mathrm{c}}$ & 6.96 & 9.61 & 8.28 \\
\hline G & 16.05 & 20.91 & 18.48 & 600.83 & 636.78 & 618.80 & $19.36^{\mathrm{ab}}$ & $20.88^{\mathrm{ab}}$ & $20.13^{\mathrm{b}}$ & 5.1 & 7.01 & 6.05 \\
\hline B & 15.54 & 18.69 & 16.93 & 576.75 & 537.00 & 556.88 & $20.82^{\mathrm{a}}$ & $22.94^{\mathrm{a}}$ & $21.89^{\mathrm{a}}$ & 4.15 & 5.5 & 4.83 \\
\hline W & 16.41 & 19.47 & 17.94 & 595.50 & 574.83 & 585.17 & $19.02^{\mathrm{ab}}$ & $20.95^{\mathrm{ab}}$ & $19.99^{\mathrm{b}}$ & 6.91 & 8.51 & 7.71 \\
\hline $\mathrm{R} \rightarrow \mathrm{G}$ & 16.26 & 22.50 & 19.38 & 695.70 & 705.39 & 700.54 & $17.77^{\mathrm{b}}$ & $19.81^{\mathrm{b}}$ & $18.83^{\mathrm{bc}}$ & 5.83 & 8.50 & 7.17 \\
\hline $\mathrm{R} \rightarrow \mathrm{B}$ & 15.75 & 18.33 & 17.22 & 714.75 & 632.28 & 673.52 & $17.84^{\mathrm{b}}$ & $20.18^{\mathrm{b}}$ & $18.98^{\mathrm{bc}}$ & 4.38 & 5.71 & 5.05 \\
\hline $\mathrm{R} \rightarrow \mathrm{G} \rightarrow \mathrm{B}$ & 16.02 & 22.59 & 19.31 & 721.50 & 507.15 & 614.33 & $17.84^{\mathrm{b}}$ & $20.66^{\mathrm{ab}}$ & $19.26^{\mathrm{b}}$ & 5.34 & 7.21 & 6.28 \\
\hline $\mathrm{R} \rightarrow \mathrm{B} \rightarrow \mathrm{G}$ & 15.75 & 19.92 & 17.84 & 659.25 & 668.28 & 663.77 & $17.34^{\mathrm{b}}$ & $19.95^{\mathrm{b}}$ & $18.46^{\mathrm{bc}}$ & 4.89 & 6.49 & 5.69 \\
\hline SEM & 0.19 & 0.63 & 0.36 & 27.91 & 29.19 & 20.67 & 0.33 & 0.34 & 0.27 & 0.78 & 0.58 & 0.53 \\
\hline \multicolumn{13}{|c|}{ Test statistics } \\
\hline $\mathrm{F}_{7,72}$ & 0.41 & 1.12 & 1.17 & 0.47 & 1.02 & 0.93 & 2.63 & 3.52 & 8.61 & 0.17 & 0.73 & 0.63 \\
\hline P Value & 0.8860 & 0.3850 & 0.3582 & 0.8461 & 0.4409 & 0.4992 & 0.0481 & 0.0113 & 0.001 & 0.988 & 0.6472 & 0.723 \\
\hline
\end{tabular}

Two pens of 10 birds each per mean, ${ }^{\text {a,b,c }}$ Mean values within a column followed by the same letter are not significantly different $(p>0.05)$; SEM, standard error; M, morning; A, afternoon; R, Red; G, green; B, blue; W, white; R $\rightarrow G$, red $\rightarrow$ green; $R \rightarrow B$, red $\rightarrow$ blue; $R \rightarrow G \rightarrow B$, red $\rightarrow$ green $\rightarrow$ blue; $\mathrm{R} \rightarrow \mathrm{B} \rightarrow \mathrm{G}$, red $\rightarrow$ blue $\rightarrow$ green.

stimulated ground pecking and scratching behaviors $(p<$ 0.05 ) compared to those in birds under B light. Both ground pecking and scratching behaviors were frequently observed during the afternoon. Furthermore, wing flapping behavior was not affected by light color but tail wagging frequency increased under $\mathrm{R}$ light compared to that under the $\mathrm{B}$ treatment. 
Table 5. Effect of LED color on different behaviors of laying hens

\begin{tabular}{|c|c|c|c|c|c|c|c|c|c|c|c|c|}
\hline \multirow{2}{*}{ Treatment } & \multicolumn{3}{|c|}{ Ground pecking (no./h) } & \multicolumn{3}{|c|}{ Ground scratching (no./h) } & \multicolumn{3}{|c|}{ Tail wagging (no./h) } & \multicolumn{3}{|c|}{ wing flapping (no./h) } \\
\hline & M & A & Mean & M & A & Mean & M & A & Mean & M & A & Mean \\
\hline $\mathrm{R}$ & 349.50 & $406.08^{\mathrm{a}}$ & $377.79^{\mathrm{a}}$ & 43.80 & $72.63^{\mathrm{a}}$ & $58.22^{\mathrm{a}}$ & 21.00 & $25.32^{\mathrm{a}}$ & $23.16^{\mathrm{a}}$ & 18.00 & 15.78 & 16.89 \\
\hline G & 315.00 & $348.18^{\mathrm{ab}}$ & $331.59^{\mathrm{ab}}$ & 44.49 & $65.64^{\mathrm{ab}}$ & $55.07^{\mathrm{ab}}$ & 14.01 & $21.75^{\mathrm{ab}}$ & $17.88^{\mathrm{b}}$ & 16.13 & 15.42 & 15.77 \\
\hline B & 231.00 & $252.78^{\mathrm{c}}$ & $248.94^{\mathrm{c}}$ & 31.5 & $51.78^{\mathrm{b}}$ & $47.45^{\mathrm{bc}}$ & 16.67 & $15.78^{\mathrm{c}}$ & $18.29^{\mathrm{b}}$ & 15.37 & 15.78 & 15.58 \\
\hline W & 342.00 & $334.53^{\mathrm{abc}}$ & $338.26^{\mathrm{ab}}$ & 36.0 & $63.60^{\mathrm{ab}}$ & $49.80^{\mathrm{abc}}$ & 17.75 & $17.13^{\mathrm{bc}}$ & $17.44^{\mathrm{b}}$ & 15.75 & 14.43 & 15.09 \\
\hline $\mathrm{R} \rightarrow \mathrm{G}$ & 327.00 & $385.08^{\mathrm{ab}}$ & $356.04^{\mathrm{a}}$ & 44.07 & $63.39^{\mathrm{ab}}$ & $47.93^{\mathrm{bc}}$ & 20.18 & $19.89^{\mathrm{bc}}$ & $20.03^{\mathrm{ab}}$ & 16.50 & 13.53 & 15.01 \\
\hline $\mathrm{R} \rightarrow \mathrm{B}$ & 255.90 & $340.08^{\mathrm{abc}}$ & $297.99^{\mathrm{abc}}$ & 36.75 & $49.89^{\mathrm{b}}$ & $43.32^{\mathrm{c}}$ & 13.50 & $17.67^{\mathrm{bc}}$ & $15.59^{\mathrm{b}}$ & 16.88 & 17.28 & 17.08 \\
\hline $\mathrm{R} \rightarrow \mathrm{G} \rightarrow \mathrm{B}$ & 313.50 & $333.75^{\mathrm{abc}}$ & $323.63^{\mathrm{abc}}$ & 42.06 & $61.68^{\mathrm{ab}}$ & $51.87^{\mathrm{abc}}$ & 21.75 & $19.38^{\mathrm{bc}}$ & $20.57^{\mathrm{ab}}$ & 18.00 & 12.72 & 15.36 \\
\hline $\mathrm{R} \rightarrow \mathrm{B} \rightarrow \mathrm{G}$ & 245.10 & $303.87^{\mathrm{bc}}$ & $262.43^{\mathrm{bc}}$ & 35.00 & $49.52^{\mathrm{b}}$ & $44.26^{\mathrm{c}}$ & 18.38 & $19.92^{\mathrm{bc}}$ & $17.08^{\mathrm{b}}$ & 15.0 & 14.28 & 14.64 \\
\hline SEM & 14.45 & 11.98 & 10.58 & 1.32 & 2.09 & 1.22 & 0.83 & 0.71 & 0.62 & 0.41 & 0.45 & 0.26 \\
\hline \multicolumn{13}{|c|}{ Test statistics } \\
\hline $\mathrm{F}_{7,72}$ & 1.40 & 2.62 & 3.18 & 1.89 & 2.92 & 3.43 & 2.16 & 3.26 & 2.45 & 0.90 & 1.41 & 1.56 \\
\hline P Value & 0.2510 & 0.0369 & 0.0158 & 0.1156 & 0.0233 & 0.0110 & 0.075 & 0.0141 & 0.0481 & 0.525 & 0.247 & 0.1958 \\
\hline
\end{tabular}

Two pens of 10 birds each per mean. ${ }^{\mathrm{a}, \mathrm{b}, \mathrm{c}}$ Mean values within a column followed by the same letter are not significantly different $(p>0.05)$; SEM, standard error, M, morning; A, afternoon; R, red; G, green; B, blue; W, white; $R \rightarrow G$, red $\rightarrow$ green; $R \rightarrow B$, red $\rightarrow$ blue; $R \rightarrow G \rightarrow B$, red $\rightarrow$ green $\rightarrow$ blue; $\mathrm{R} \rightarrow \mathrm{B} \rightarrow \mathrm{G}$, red $\rightarrow$ blue $\rightarrow$ green.

\section{Discussion}

Our results show that birds reared under monochromatic $\mathrm{R}$ light increased egg production. This finding is similar to that reported by Kim et al. (2012). Rozenboim et al. (1998) reported that birds reared under $\mathrm{R}$ light mature earlier and show higher egg production. Furthermore, light color switched from monochromatic $\mathrm{R}$ to a combined $\mathrm{R} \rightarrow \mathrm{G}$ treatment stimulates the reproductive axis, enhances follicular growth, and, thus, increases egg production. These results confirm the recent findings of Hassan et al. (2013) who found that $\mathrm{R} \rightarrow \mathrm{G}$ treatment increases serum follicle stimulating hormone and $17 \beta$-estradiol concentrations. These hormones enhance the growth and number of ovarian follicles, which may be associated with the rate of egg production. Consistent with the present results, Rani et al. (2001) conducted an experiment on blackhead bunting and reported that 10L:14D and 14L:10D with $4 \mathrm{~h}$ of $\mathrm{G}$ light significantly increases testicular growth, and this mechanism may also be effective in laying hens. In contrast, Mobarkey et al. (2010) indicated that retinal photostimulation with $\mathrm{G}$ light combined with non-photostimulatory $\mathrm{R}$ light reduces egg production in broiler breeder hens. This difference is due to the duration of time exposed to the light combinations.

Egg weight increased under the $\mathrm{G}$ and $\mathrm{B}$ light treatments due to a decrease in the rate of egg production. However, $G$ and B light stimulated growth and enhanced body size and, thus, increased egg weight, which corroborated the findings of Hassan et al. (2013). Similar results were also obtained by Kim et al. (2012) and Pyrzak et al. (1987) and suggest that a difference in egg weight is a consequence of the rate of egg production rather than the lighting treatment. But, in another experiment, either W light (Er et al., 2007) and W or B light color enhances egg weight in laying hens (Kim et al., 2010). This variation might be due to variations in egg production. Mean feed conversion ratio (FCR) improved under the $\mathrm{R} \rightarrow \mathrm{G}$ light treatment, which may have been due to the sensitivity of the light wavelengths and thus reflected by a significant increase in egg production that influenced the mean FCR of laying hens.

Egg shell breaking strength was significantly higher in birds under the $G$ treatment, which may have been due to growth hormone enhanced calcium absorption in the small intestine (Fleet et al., 1994) or due to lower surface to volume ratio of relatively wider eggs resulting in relatively less weight loss due to evaporative loss, which corresponded with previous findings (Er et al., 2007; Pyrzak et al., 1987). Shell color was also influenced by the $R \rightarrow G \rightarrow B$ and $R \rightarrow B$ $\rightarrow \mathrm{G}$ treatments, which may have been due to the combined light enhancing effect on the epithelial cells lining the surface of the shell gland and synthesis and accumulation of biliverdin-IX pigments (Butcher and Miles, 2003).

Feather pecking behavior was not significantly affected by light color but a numerically higher frequency found in birds under the $\mathrm{R}$ color may have been due to hypothalamically active $\mathrm{R}$ light directly affecting the pineal gland, which may have stimulated interactive behavior and increased feather pecking frequency. Similar findings were reported for broilers (Prayitno et al., 1997; Heshmatollah, 2007; Sultana et al., 2013). Those studies reported that birds are more aggressive and more active under $\mathrm{R}$ light. In another study Schumaier et al. (1968) reported that $\mathrm{R}$ light reduces cannibalism, due to the bird's inability to see the blood on injured birds. Consequently, the higher activity observed in the afternoon was probably due to the laying hens spending their morning time seeking a nest and laying eggs. Clearly, an increase in the frequency of one behavior will necessitate a decrease in another behavior within the time budget (Sultana et al., 2013).

Although preening behavior was not significantly affected by the lighting treatments, the majority of the bird's time was involved in preening due to its less aggressive nature. Birds 
preferred to perch under the $\mathrm{B}$ light compared to under the $\mathrm{R}$ lighting conditions. It is reasonable to assume that under $\mathrm{B}$ light birds spent more inactive time sitting on the perch, whereas birds illuminated with $\mathrm{R}$ light were more active and engaged in more ground pecking and scratching behaviors. These behaviors might be due to higher visual sensitivity of the birds under $\mathrm{R}$ compared with under the $\mathrm{B}$ end of the spectrum (Hartwig and Veen, 1979; Prayitno et al., 1997; Lewis and Morris, 2000). These behaviors were also motivated due to the higher number of social interactions under $\mathrm{R}$ light. We also observed that ground scratching was associated with ground pecking behavior. Birds frequently scratched the litter with their claws and pecked the ground, and these observations confirm the findings of Keeling and Jensen (1995). Those authors observed that active birds performed more ground pecking behavior. In contrast, Savory et al. (1999) found that birds involved with feather pecking decrease the rate of ground pecking behavior. Wood-Gush, (1987) reported that $\mathrm{R}$ light increases tail wagging frequency compared to that of B light, which is consistent with the present results and may be due to the visibility of companion birds under longer wavelengths. Banks and Koen, (1989) found that the wing flapping behavior is more common in frustrated or conflicted birds and is expressed in the corner of the pen. We found that wing flapping behavior was not affected by either the monochromatic or the combined light color. Our results provide clear indications that light color influences the performance and behavior of laying hens.

In conclusion, our results indicate that Hy-line brown laying hens reared under combinations of monochromatic lights ( $14 \mathrm{~h} \mathrm{R}$ and $2 \mathrm{~h}$ G light daily) had higher egg production and better feed conversion efficiency. Moreover, the $\mathrm{R}$ light treatment increased ground pecking, ground scratching, and tail wagging behaviors, whereas B light decreased these behaviors and more birds perched. Therefore, the combined light colors did not adversely affect the bird's behavioral needs. A combination of LED light colors $(\mathrm{R} \rightarrow \mathrm{G})$ would be comparable with monochromatic $\mathrm{R}$ light and could be applied to increase egg production in laying hens.

\section{References}

Banks PA and Koen TB. Intermittent lighting regimens for laying hens. Poultry Science, 68: 739-743. 1989.

Butcher GD and Miles RD. Factors causing poor pigmentation of brown shelled eggs. University of Florida. 2003.

Er D, Wang Z, Cao J and Chen Y. Effect of monochromatic light on the egg quality of laying hens. Journal of Applied Poultry Research, 16: 605-612. 2007.

Fleet JC, Bruns ME, Hock JM and Wood RJ. Growth hormone and parathyroid hormone stimulate intestinal calcium absorption in aged female rats. Endocrinology, 134: 1755-1760. 1994.

Hassan MR, Sultana S, Choe HS, Ryu KS. Effect of monochromatic and combined light colour on performance, blood parameters, ovarian morphology and reproductive hormones in laying hens.
Italian Journal of Animal Science, 12: 359-364. 2013.

Hartwig HG and Veen T. Spectral characteristics of visible radiation penetrating into the brain and stimulating extraretinal photoreceptors. Journal of Comparative Physiology A, 130: 277-282. 1979.

Keeling L and Jensen P. Do feather pecking and cannibalistic hens have different personalities? Applied Animal Behaviour Science, 44: 257-281. 1995.

Khosravinia H. Preference of broiler chicks for color of lighting and feed. Journal of Poultry Science, 44: 213-219. 2007.

Kim MJ, Hossian MS, Nazma A, Jae CN, Han TB, Hwan KK, Dong WK, Chae HC, Choi HC and Ok SS. Effect of monochromatic light on sexual maturity, production performance and egg quality of laying hens. Avian Biology Research, 5: 69-74. 2012.

Kim MJ, Choi HC, Ok SS, Chae HS and Jae CN. Study of different sources and wavelengths of light on laying egg characteristics in laying hens. Korean Journal of Poultry Science, 37: 383388. 2010.

Lewis PD and Morris TR. Poultry and colored light. World's Poultry Science Journal, 56: 189-207. 2000.

Mobarkey N, Avital N, Heiblum R and Rozenboim I. The role of retinal and extra-retinal photostimulation in reproductive activity in broiler breeder hens. Domestic Animal Endocrinology, 38: 235-243. 2010.

Prayitno DS, Phillips CJC and Stokes DK. The effects of color and intensity of light on behavior and leg disorders in broiler chickens. Poultry Science, 76: 1674-1681. 1997.

Pyrzak R, Snapir N, Goodman G and Perek M. The effect of light wavelength on the production and quality of eggs of the domestic hen. Theriogenology, 28: 947-960. 1987.

Rani S, Singh S, Misra M, Kumar V. The influence of light wavelength on reproductive photorefractoriness in migratory blackheaded bunting (Emberiza melanocephala). Reproduction Nutrition Development, 41: 277-284. 2001.

Rozenboim I, Zilberman E and Gvaryahu G. New monochromatic light source for lying hen. Poultry Science, 77: 1695-1698. 1998.

SAS Institute Inc. SAS/STAT software changes and enhancements through release 9.0. Cary, North Carolina: SAS Institute. 1996.

Savory CJ, Mann JS and MaCleod MG. Incidence of pecking damage in growing bantams in relation to food form, group size, stocking density, dietary tryptophan concentration and dietary protein source. British Poultry Science, 40: 579-584. 1999.

Schumaier G, Harrison PC and McGinnis J. Effects of colored fluorescent light on growth, cannibalism and subsequent egg production of single comb white leghorn pullets. Poultry Science, 47: 1599-1602. 1968.

Sultana S, Hassan MR, Choe HS and Ryu KS. The effect of monochromatic and mixed LED light colour on the behaviour and fear responses of broiler chicken. Avian Biology Research, 6: 207-214. 2013.

Steel RGD and Torrie JH. Analysis of Covariance. Principles and Procedures of Statistics: A Biometrical Approach. McGrawHill, New York, PP. 401-437. 1980.

In: Cognitive Aspects of Social Behavior in the Domestic Fowl (Zayan R and Duncan IJH Eds.). pp. 34-39. Elsevier. Amsterdam. 1987. 